
\title{
25 Research Square \\ Solving Fokker-Planck equations using deep KD-tree with a small amount of data
}

\author{
Yong Xu ( $\nabla$ hsux3@nwpu.edu.cn ) \\ Northwestern Polytechnical University https://orcid.org/0000-0002-8407-4650 \\ Hao Zhang \\ Northwestern Polytechnical University \\ Qi Liu \\ Northwestern Polytechnical University \\ Xiaolong Wang \\ Shaanxi Normal University \\ Yongge Li \\ Northwestern Polytechnical University
}

\section{Research Article}

Keywords: Fokker-Planck equation , deep KD-tree , deep learning , super-DL-FP

Posted Date: January 31st, 2022

DOI: https://doi.org/10.21203/rs.3.rs-1265549/v1

License: (c) (1) This work is licensed under a Creative Commons Attribution 4.0 International License. Read Full License 


\title{
Solving Fokker-Planck equations using deep KD-tree with a small amount of data
}

\author{
Hao Zhang ${ }^{1,2}$. Yong $\mathrm{Xu}^{1,3}{ }^{*} \cdot \mathrm{Qi}$ Liu \\ 1 . Xiaolong Wang ${ }^{4}$. Yongge Li ${ }^{1}$
}

Received: date / Accepted: date

\begin{abstract}
The Fokker-Planck (FP) equation can deterministically describe the evolution of the probability density function, which plays an extremely significant role in the fields of stochastic dynamics. Unfortunately, the limited samples that arise from the consideration of engineering practice are inevitable, which restricts the solving of the FP equation. Accordingly, in the present study, a super-DL-FP framework is established to solve the steady-state FP equation with a small amount of data, through combining the deep KD-tree and the DL-FP approach proposed in [Chaos 30, 013133 (2020)]. It should be emphasized that the normalization condition is of great importance and have to be considered in solving the steady-state FP equation. An appropriate integral estimation for the normalization condition under non-uniform meshing can effectively improve the precision of the solution, but it is still a challenging problem, especially for the case of small data. Thus, the so-called deep KDtree method is innovatively proposed to estimate the normalized integral with a small random dataset. The main target is to obtain the appropriate discrete integral points and corresponding integral volumes by executing multiple KDtree segmentation based on random data on the integral region. Several numer-

$凶$ Yong $\mathrm{Xu}$

E-mail: hsux3@nwpu.edu.cn

1 School of Mathematics and Statistics, Northwestern Polytechnical University, Xi'an 710072, China.

2 Department of Engineering Mechanics, Northwestern Polytechnical University, Xi'an 710072, China

3 MIIT Key Laboratory of Dynamics and Control of Complex Systems, Northwestern Polytechnical University, Xi'an 710072, China.

4 School of Mathematics and Statistics, Shaanxi Normal University, Xi'an 710119, China.
\end{abstract}


ical experiments and comparisons are implemented to illustrate the superior performance of the super-DL-FP method. The obtained results indicate that the proposed algorithm can accomplish higher accuracy in the sense of lower cost than the well-known algorithms like center difference scheme, Chebyshev spectrum algorithm, and normalized flow approach.

Keywords Fokker-Planck equation · deep KD-tree · deep learning · super-DL-FP

\section{Introduction}

The Fokker-Planck (FP) equation can describe the evolution for the distribution of fluctuating macroscopic variables in stochastic dynamics [19]. The application of the FP equation is not restricted to microscopic systems such as thermal equilibrium [6], bioscience [31,16], and chemistry [3], but also macroscopic systems such as economics [8] and engineering [25]. By solving the FP equation, one obtains distribution functions from which any averages of macroscopic variables are obtained by integration. Although the FP equation is essentially a second-order parabolic partial differential equation, it is challenging to solve it theoretically [19]. The present research on the numerical solution to the FP equation is limited by meshing. Especially for the steadystate FP equation with natural boundary, all the discrete points need to be involved in computation as the emergence of normalization condition. The number of calculation points increases exponentially with the increase of dimension, which lead to huge amount of calculations. Besides, in engineering practice, no enough experimental points are available to meet the computing requirements in general. Therefore, this paper mainly focuses on how to reduce the need for calculation points.

The discretization methods for solving the FP equations are strongly depended on meshing, resulting in an exponential increase in computation with dimension, for instance, finite element $[17,9]$, finite difference $[13,20]$, path integral $[5,26]$. In particular, it must make a trade-off between precision and hardware storage capacity when dealing with high-dimensional cases. Meanwhile, recalculation or interpolation have to be done in solving the values on the off-grid points as only the points on the grid can be obtained. The Monte Carlo simulation $[24,22]$ is also a common approach for solving FP equations, which is a simple and efficient tool for solving one- and two-dimensional probability density function (PDF). However, this approach needs to consume massive samples and is not feasible with the dimension increases.

Recently, to solve the grid dependency problem, the neural networks (NNs) are proposed to solve differential equations, even the steady-state FP equation with natural boundary $[27,4,28,29,18,10,21,15,30,23]$. However, the huge amount of calculation data is still required. The $\mathrm{NN}$ can calculate the derivate operator analytically, which allows the only local information to be considered. To satisfy the normalization condition, it's necessary to consider the global information. Aiming at the normalization condition in FP equation, 
the grid discretization scheme is adopted [27], which makes the training data grow exponentially with the increase of dimension. Another approach is to use the PDF calculated from prior data to supervise the solution of the FP equation instead of the supervision of normalization condition $[4,28]$. However, the reasonable prior distribution is not easily obtained without enough available data. Furthermore, the number of training data is an important factor affecting the speed of $\mathrm{NN}$, which determines the depth of the network and the number of parameters. Therefore, reducing the need for training data is an imperative issue, which is benefit to improve the calculation speed and increase the dimension for solving the FP equations.

Both the traditional discretization methods and the NN-based approaches strongly depend on a large amount of data, whereas the problem of small samples are inevitable and more desired in the practical applications. Accordingly, the present study attempt to develop an effective technique for solving the steady-state FP equation based on small datasets. We notice that the NN can compute derivatives in analytical form, which means that the derivative of one point does not depend on other points. Hence, the random points can be adopted as calculation points. Meanwhile, the higher dimensional distribution function is sparse, which means that a small number of points can be used to estimate the integral of PDF. Thus, as long as the number of basic training points is satisfied, the solution can be completed by using NN. However, it also brings up a key question which is how to calculate the integral for these finite stochastic experimental data points with appropriate geometric volume. To solve this problem, we propose a deep KD-tree algorithm constructed by multi-layer KD-tree, which can adaptively and efficiently obtain the corresponding integral volume for random training points, and the separated boundary points for the boundary condition. Through combining with the DL-FP framework [27] proposed in our previous work, the super-DL-FP algorithm is proposed to make it possible to solve the FP equation with a small amount of data.

The structure of this paper is as follows. Sec. II gives a detailed description of the algorithms including KD-tree, deep KD-tree and the super-DL-FP approach. In Sec. III, three examples consisting of 2D, 4D and 6D systems are shown to demonstrate the super-DL-FP can be used successfully. At the same time, we compare the proposed algorithm with other approaches including difference schemes and normalized flow algorithm to illustrate the superiority of the super-DL-FP. In the last section, conclusions are presented to close this paper.

\section{Methodology}

In this section, three sub-sections are shown to explain the proposed algorithm. The FP equation and the necessary conditions for its solution will be described in detail in Sect. 2.1. In Sect. 2.2, the deep KD-tree algorithm constructed by multi-layer KD-tree will be elaborated at length and the algorithm flow will be 
given. We will introduce how to combine deep KD-tree and DL-FP algorithm to build super-DL-FP scheme in Sect. 2.3, and give a detailed algorithm flow framework.

2.1 The Langevin equation and the Fokker-Planck equation

For $N$ variables $x=x_{1}, x_{2}, \ldots, x_{N}$ the general Langevin equations have the form $(i=1,2, \ldots, N)$

$$
\dot{x_{i}}=h_{i}(x, t)+g_{i j} \Gamma_{j}(t),
$$

where $\Gamma_{j}(t)$ are Gaussian random variables with zero mean and with correlation functions proportional to the $\delta$ function, i.e. $\left\langle\Gamma_{i}(t)\right\rangle=0$ and $\left\langle\Gamma_{i}(t) \Gamma_{j}\left(t^{\prime}\right)\right\rangle=$ $2 \delta_{i j} \delta\left(t-t^{\prime}\right)$, where $\langle\cdot\rangle$ represents the expectation.

The corresponding FP equation for the probability density $p(x, t)$ is

$$
\begin{gathered}
\frac{\partial p(x, t)}{\partial t}=\mathcal{L}_{F P} p(x, t) \\
\mathcal{L}_{F P}=-\frac{\partial}{\partial x} D^{(1)}(x, t)+\frac{\partial^{2}}{\partial x^{2}} D^{(2)}(x, t),
\end{gathered}
$$

where $\mathcal{L}_{F P}$ is the FP operator. Under the definition of Itô integral, $D^{(1)}(x, t)=$ $h(x, t)$ represents the drift coefficient, and $D^{(2)}(x, t)=g^{2}(x, t)$ is the diffusion coefficient.

For a stationary process the probability current must be zero, then the stationary FP equation is

$$
\mathcal{L}_{F P} p(x, t)=0 .
$$

When considering the natural boundary conditions $\left(x_{\min }=-\infty, x_{\max }=\right.$ $\infty)$, the probability $p(x)$ vanishes at $x= \pm \infty$ and then guarantees that the normalization is perserved

$$
\int_{x_{\min }}^{x_{\max }} p(x) d x=1 .
$$

In summary, four conditions should be considered when solving the stationary FP equation. (i) Satisfying the format of FP equation. (ii) The probability at the boundary is zero. (iii) Satisfying the normalization condition. (iv) The $\mathcal{W}(x)$ is non-negative. It is just a regularization constraint that can be satisfied as long as the assumed solution is always positive for (iv). As for the other conditions, it is easy to fall into trivial solutions if we only focus on (i) and (ii). Therefore, normalization condition (iii) should never be ignored. When discretized numerical method is used to solve the steady-state FP equation, normalization conditions as a limiting factor make it necessary to utilize all discrete data, which inevitably makes the amount of the computation points rise exponentially with the increase of dimension. Here, we mainly consider using a small amount of random data instead of the whole to solve the FP 
equation with NN. But along with this comes a problem, that is how to calculate the normalized value for these random points. At this point, the key problem is to match the appropriate integral volume for these random points. Therefore, the deep KD-tree is proposed to solve this problem which will be introduced at length in Sect. 2.2.

\subsection{Deep KD-tree algorithm}

When calculating integrals numerically, a very simple approach is to discretize the integral which can be expressed as

$$
\int_{x_{\min }}^{x_{\max }} p(x) d x \approx \sum_{i=1}^{N_{\mathrm{all}}} p\left(x_{i}\right) v_{i},
$$

where $x_{i}$ is the selected integral point, $v_{i}$ is the corresponding integral volume, and $N_{\text {all }}$ represents the number of the selected integral points. The general way to select points is to take points at equal intervals, or some points obtained by polynomial approximation integration, such as the Gauss-Legendre points. At this time, it is easy to get $v_{i}$ which can be seen as the volume or the weight of $p\left(x_{i}\right)$. Nevertheless, it is imperative to select an appropriate segmentation approach to match the corresponding integral volume for these points if the integral points are random experimental points. A very naive idea is whether the KD-tree algorithm [2] could be directly used to divide the integral volume for these random points, inspired by the work [7] which applies the KD-tree splitting the volume of the MCMC points to accelerate the Vegas integral method. Three questions should be considered. What is the KD-tree method and how to apply it? Whether the training data and boundary data required by DL-FP algorithm can be obtained from the KD-tree approach? Whether the volume divided by the KD-tree can be utilized directly? The deep KD-tree method proposed by us will be provided in the process of responding to the three questions.

What is the KD-tree method and how to apply it? The working principle of KD-tree is as follows, the median of the dimension with the largest variance is selected as the parent node, and then the remaining points are divided to both sides as the child nodes based on the selected dimension by making a straight line perpendicular to the hypercube. Then the dividing method is repeated for the points on both sides until all points are divided. This leads to the problem that the points being divided are basically on the boundary of the partition volume. When the number of points is large enough, one can estimate the integral by taking the points as integral points and multiplying them by the corresponding volume, but when the number of points is small, this calculation becomes problematic. For example, if a high value is chosen where the fluctuation of the function is relatively large, the calculated integral will be incorrect. A conservative approach would be to pick the midpoint of the partition volume as the integral point, which works like the trapezoidal 
integral formula. Therefore, after KD-tree segmentation, the midpoint of the divided volume should be used as the position of the integral point.

Whether the training data and boundary data required by DL-FP algorithm can be obtained from the KD-tree approach? The training set and boundary set are required when training the FP equation using the DL-FP algorithm. The random points generated by experiments can roughly reflect the distribution function. The probability of points close to geometric boundaries is very small, so they can be used as boundary points for training. When KD-tree is used for partition, the segmented hypercube corresponding to the boundary points at geometric positions must have a dimension that is the boundary of the region to be calculated. Hence, boundary points can be extracted from the divided points. It should be noted that, according to the answer to the first question, the midpoint of the partitioned hypercube is actually used as the integral point, so the points on the boundary will expand out, which is more suitable as the boundary point.

Then, the algorithm flow of KD-tree is given based on the above two questions which is shown in Algorithm 1. The Algorithm 1 is mainly used to obtain volume segmentation of random points and the corresponding geometric midpoints. Different from the classical KD-tree [2] approach, we modify the output form of data without caring about the structure of the generated tree.

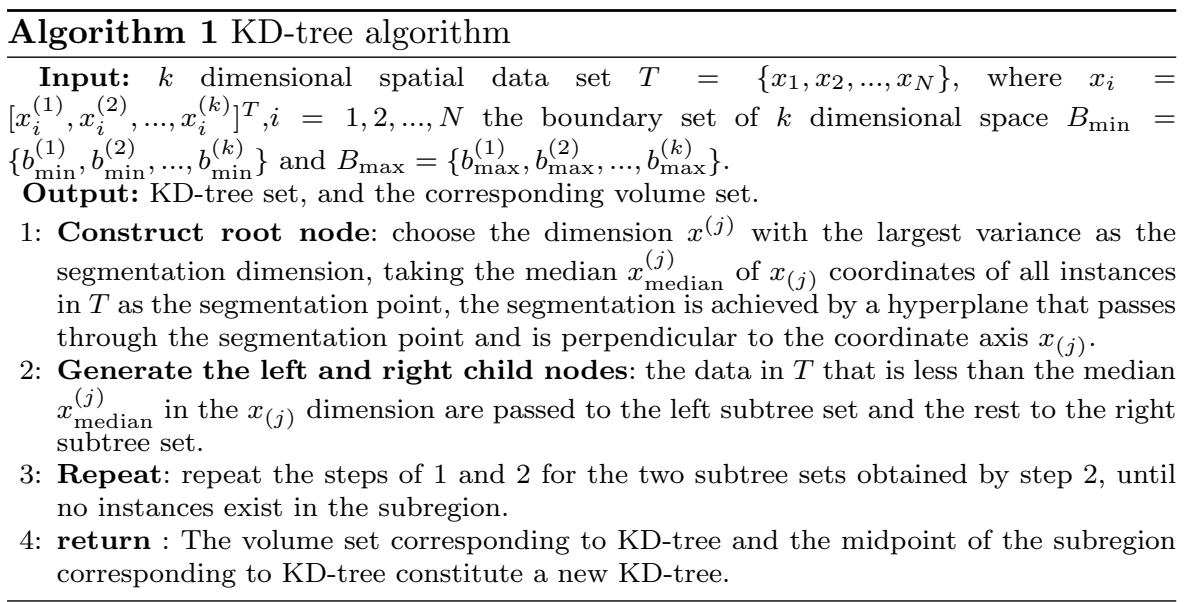



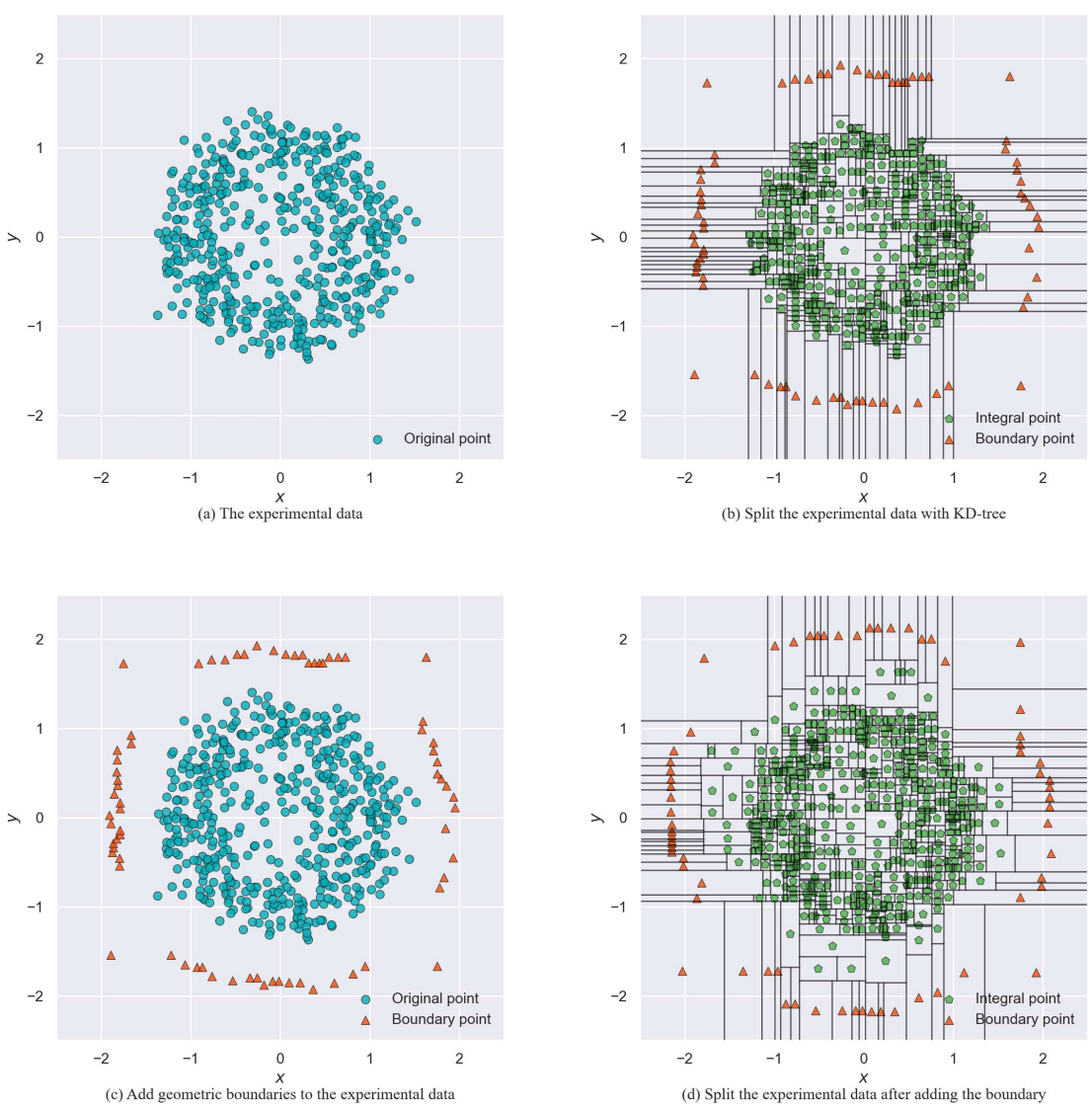

Fig. 1 (a) 500 sample points from the limit cycle distribution. (b) The KD-tree method is used to segment the data in region $[-2.5,2.5]^{2}$, and all the points are the midpoint of each small volume after segmentation. (c) Merge data, the points are a combination of the original points in (a) and the boundary points in (b). (d) After combining the data, KD-tree is used to segment the region again to obtain the integral points and boundary points where the green ones are the integral points and the orange ones represent the boundary points.

Whether the volume divided by the KD-tree can be utilized directly? To better answer the question, two-dimensional random experimental data from limit cycle oscillation is used for illustration, as shown in Fig. 1. When KD-tree is used to segment the integral hypercube, the volume of the points near the integral boundary will be relatively large, which can be seen from the region covered by the orange points in Fig. 1(b). When the DL-FP framework is used for training with all the points, a possible situation is that the NN will obtain an unpromising solution where all the probability masses are assigned to the boundary points while the probabilities of the important integral points, i.e. the green points in Fig. 1(b), are zero. Thus, the volume 
segmentation obtained by using KD-tree cannot be directly used to estimate the integral. The boundary points in Fig. 1(b) are obtained from the expansion in Fig. 1(a). But the region covered by the boundary points can not be deleted directly, because there are integrals available in the region covered by the boundary points. In particular, the integral points occupy a smaller integral region with the increase of dimension. The process is similar to peeling the skin off a fruit, leaving behind the compact structure inside. Therefore, we need to expand the area in the middle. The points shown in Fig. 1(c) are formed by combining the orange points in Fig. 1(b) and the original data in Fig. 1(a). Then the points in Fig. 1(c) are segmented by KD-tree approach, and the segmentation in Fig. 1(d) can be obtained. By comparing Fig. 1(d) with Fig. 1(b), it can be found that the effective integral region covered by the green points in the middle has increased. This expansion can be done multiple times as the dimensions increase. The specific process is as follows. The boundary points are separated by KD-tree once, then combined with the original data, and then iterating the KD-tree algorithm for the combined data from last iteration. The termination condition of iteration is to set the data ratio coefficient $\gamma$, where $0<\gamma \leq 1$. We name this method deep KD-tree which is shown in Algorithm 2. This process is equivalent to stretching the peeled fruit skin and then covering the original fruit. When KD-tree is performed again, more integral points with compact integral volume structure can be obtained. In other words, this process can also be regarded as matching suitable geometric boundaries and corresponding integral volume segmentation for random points.

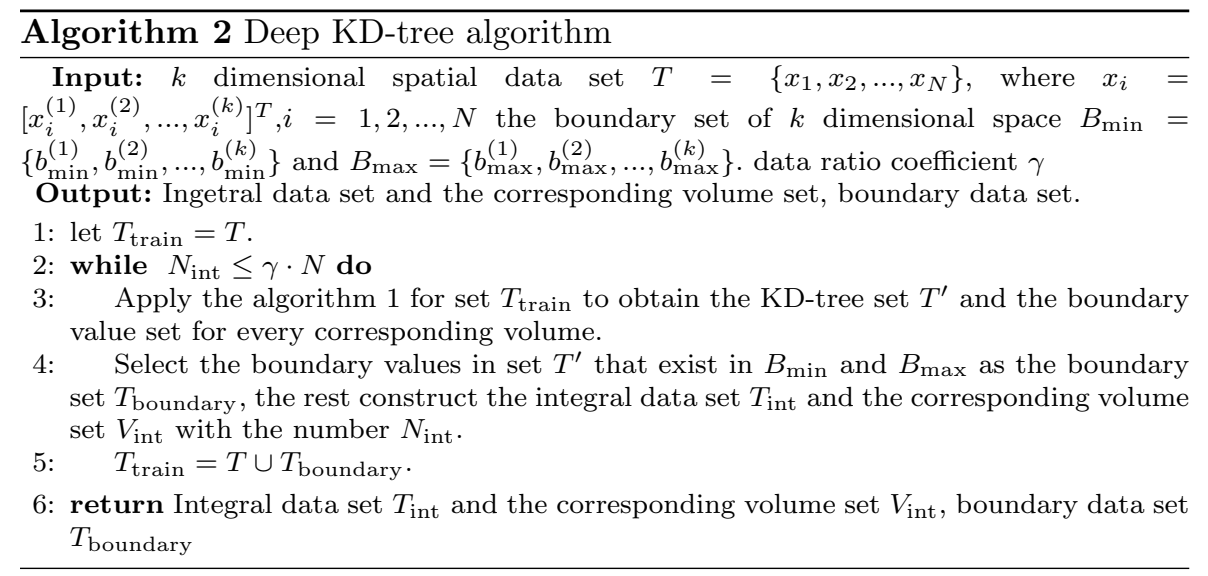

Remark 1: As a general rule, the entire integral space should be covered when computing integrals, but the deep KD-tree algorithm ignores the integral space at the boundary when dealing with integrals. In fact, the volume segmentation based on random points is not uniform, which leads to a small volume of segmentation at dense points and a large volume of sparse points 
near the boundary. Consequently, it is easy to fall into the local minimum value when training the NN. A very likely case is one where normalization is satisfied locally on the boundary and zero at other points. In order to avoid this problem, it is better to directly omit the boundary part, and the integral contribution of the boundary part is very small, so it does not matter much. Meanwhile, although the deep KD-tree method cannot make the approximate integral result one, the analytic result obtained by the $\mathrm{NN}$ can be used for analytical processing. In order to make the final result standardized, the Vegas integral technology [14] can be used to complete the normalization.

\subsection{Super-DL-FP algorithm}

With the integral point data set, corresponding integral volume set and boundary set provided by deep KD-tree, it is easy to complete the training with the aid of DL-FP framework, then the super-DL-FP algorithm is proposed. Similar to the DL-FP algorithm, the NN is still used to construct an experimental solution, and supervise the training from three perspectives. The trial solution should satisfy the form of the equation. The trial solution should meet the normalization condition, as well as fulfill the boundary condition. It should be noted that the PDF is non-negative, it only needs to add a softPlus function to the last layer of the NN to ensure that the output is positive. However, different from the DL-FP algorithm, the form of the loss function is adjusted

$\mathcal{L}=\sum_{i=1}^{N_{D}+N_{B}}\left|\mathcal{L}_{F P}\left(\widehat{p}\left(X_{i}^{D \cup B} \mid \theta_{j}\right)\right)\right|^{2}+a_{2} \cdot\left|\sum_{i}^{N_{D}} \Delta v_{i}^{D} \cdot \widehat{p}\left(X_{i}^{D} \mid \theta_{j}\right)-1\right|^{2}+\sum_{i=1}^{N_{B}}\left|\widehat{p}\left(X_{i}^{B} \mid \theta_{j}\right)\right|^{2}$,

where $\widehat{p}\left(X_{i} \mid \theta_{j}\right)$ represents the trial solution constructed by NN with the parameters $\theta_{j}$, and $a_{2}$ is the penalty factor. The supervision of equation and boundary is no longer in the form of MSE in $\mathcal{L}$, but in the form of addition. The reason for this is to increase the supervision of every value in the equation and boundary points when the number of training points is comparatively small, so as to ensure the correctness of every training point. Besides, different from the punishment used in DL-FP algorithm, the penalty factor is only added to the normalized condition. The deep KD-tree method is an approximate integral, so its supervision characteristics need to be weakened in the normalized part, and its appearance is more concerned with preventing the final training from falling into the trivial solution. At the same time, we also want to make the operation of penalty factor easier during training. The algorithm flow of super-DL-FP is shown in Algorithm 3. 


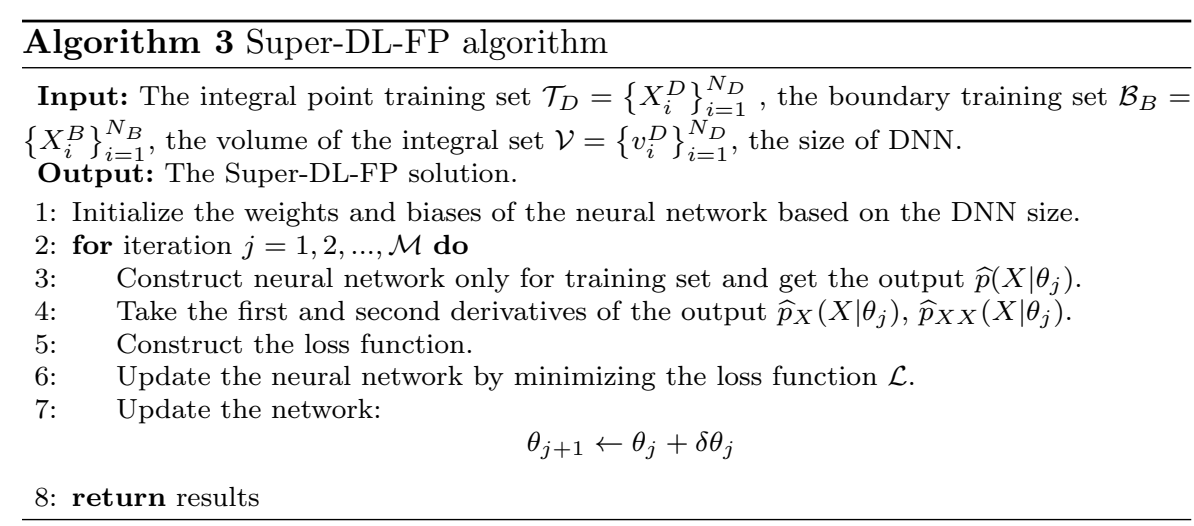

Remark 2: For the training data, the Langevin equation and FP equation exist at the same time in most cases, and the steady-state random points generated by Eq. 1 can be used as experimental points. The method of Moment Networks [12] can be used to obtain approximate FP equation of neural network when only experimental data exists and FP equation is missing.

\section{Numerical results}

In this section, three numerical evidences are provided to support our conjectures and to demonstrate that our approach can be used successfully. In the first example, we will illustrate the advantage of super-DL-FP scheme in terms of computing points compared with other classical algorithms. Furthermore, we will explain how deep KD-tree works in practice and display the correctness of the results through the second and the third examples.

\subsection{Example 1}

Consider the Van der Pol system excited by Gaussian white noise as

$$
\ddot{x}+\eta\left(-1+x^{2}+\dot{x}^{2}\right) \dot{x}+x=\sigma \Gamma,
$$

where $\Gamma$ represents a standard Gaussian white noise with the noise intensity $\sigma$. Let

$$
\left\{\begin{array}{l}
\dot{x}=y \\
\dot{y}=-\eta\left(-1+x^{2}+y^{2}\right) y-x+\sigma \Gamma .
\end{array}\right.
$$

Then the corresponding stationary FP equation is

$$
-\frac{\partial}{\partial x}[y p(x, y)]-\frac{\partial}{\partial y}\left[\left(-\eta\left(-1+x^{2}+y^{2}\right) y-x\right) p(x, y)\right]+\frac{\sigma^{2}}{2} \frac{\partial^{2}}{\partial y^{2}} p(x, y)=0
$$


and the exact steady-state solution is

$$
p_{s}(x, y)=C \cdot \exp \left[-\frac{\eta}{\sigma^{2}}\left[\left(x^{2}+y^{2}\right)-\frac{1}{2}\left(x^{2}+y^{2}\right)^{2}\right]\right],
$$

where $C$ is the normalization constant. This system can be regarded as a twodimensional system. The PDF solution will take on the shape of a crater with the parameters $\eta=1$ and $\sigma^{2}=0.3$. The training data should be prepared first when applying the super-DL-FP. The solution of Eq. 9 obtained by SRKII [11] method is taken as the training points instead of real experimental points in this example, and 100, 200 and 1000 position points are taken respectively. For these three cases, the deep KD-tree method is used to obtain experimental points where the data ratio coefficient $\gamma=1$. After obtaining the set of integral values, the corresponding set of volumes, and the boundary set, all the data is fed into the super-DL-FP framework. The penalty factor of normalized supervision is $a_{2}=0.01$. The structure of NN is constructed with three hidden layers and the number of nodes in each hidden layer is 20 . The analytic results of the NN model can be obtained after training 10,000 times, then the analytic model is normalized again by using Vegas integral algorithm. The reason for doing this is that $\mathrm{NN}$ has the advantage of being able to express the results analytically. At the same time, deep KD-tree is an approximate integral, which cannot guarantee the normalized result after training. In order to ensure that the final output PDF is standardized, the Vages are used to normalize it. The results after training are shown in Fig. 2, and the results show that the accuracy of calculation is $84.21 \%$ under only 100 experimental points, $95.24 \%$ under 200 experimental points, and 99.30\% under 1000 experimental points.
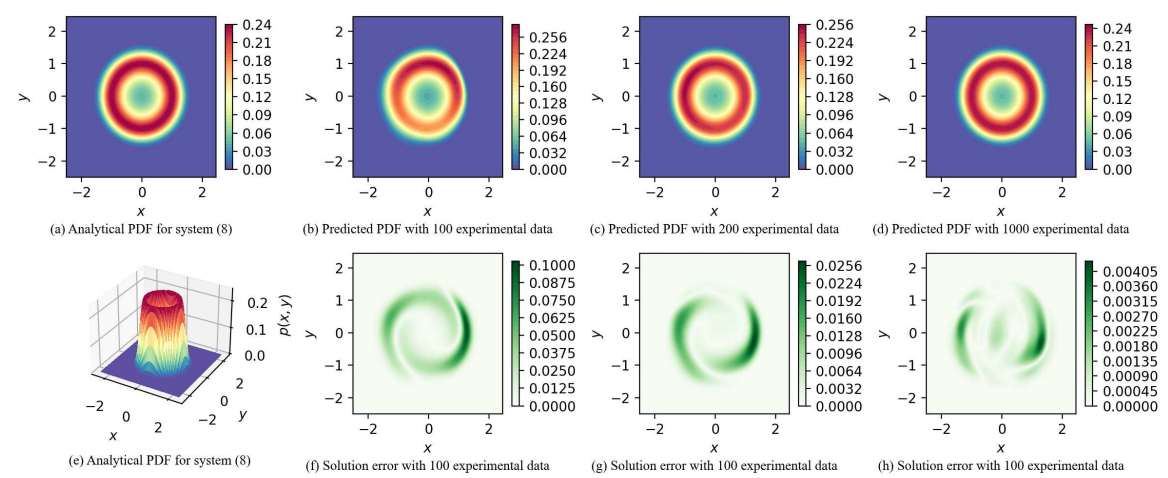

Fig. 2 (a) and (e) The analytic solution for system (8) with $\eta=1.0, \sigma^{2}=0.3$. (b) The super-DL-FP solution with 100 experimental data. (c)The super-DL-FP solution with 200 experimental data. (d) The super-DL-FP solution with 1000 experimental data. (f-h) The absolute error between analytic solution and super-DL-FP solution.

To illustrate the necessity of deep KD-tree method, the comparison between the data sets generated by deep KD-tree and KD-tree directly is analyzed, and 
the results are shown in Fig. 3(a). The red dots and lines represent the integral contribution rate $R_{\text {int }}$ defined as

$$
R_{\mathrm{int}}=\frac{\sum_{i=1}^{N_{\mathrm{int}}} p\left(x_{i}\right) \cdot v_{i}^{\prime}}{1} \cdot 100 \%,
$$

where $p(\cdot)$ is the analytical PDF of Eq. $10, N_{\text {int }}$ represents the number of integral points and $v_{i}^{\prime}$ is the corresponding integral volume. Two important conclusions can be drawn from the results. The first one is that the deep KD-tree method can greatly improve the integral contribution rate, as the deep KD-tree method can allow more experimental points to enter the integral point set and match appropriate integral volume for them. The second is that the accuracy of integration is positively correlated with the accuracy of training, which further illustrates the importance of ensuring the normalization conditions in solving FP equations. Normalization is an important factor in determining whether the PDF shape can be formed during training.

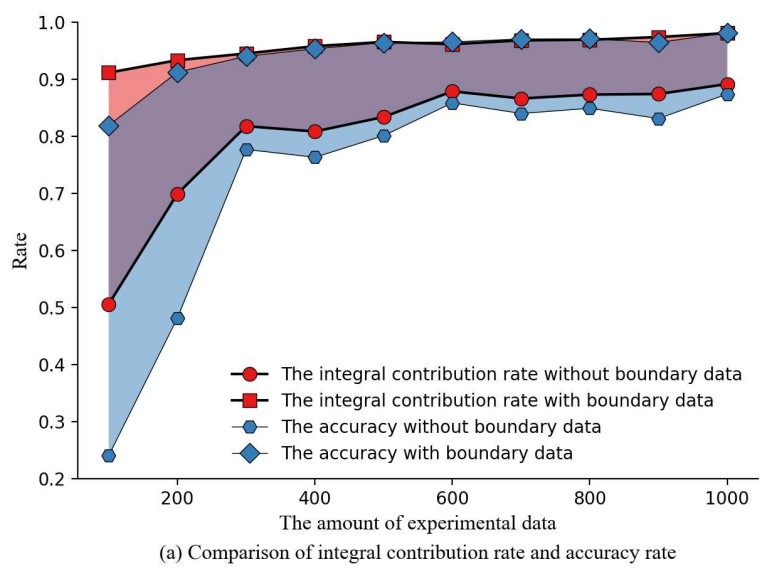

Fig. 3 Comparison of integral contribution rate and data utilization rate.

To further illustrate the value of this work, the results of super-DL-FP were compared with the central difference (CD), Chebyshev spectrum (CP), and Sylvester flow (SF) method [1]. Here, 200 calculation points are selected for different algorithms, as shown in Fig. 4, it can be obviously found that the result of the super-DL-FP scheme is more accurate and smooth. It should be emphasized here that the super-DL-FP obtains the solution in analytical form, which can be used for further analytical calculation, while the Chebyshev spectrum gains value at the point of calculation, which is difficult to be used for further analytical processing. Center difference and Chebyshev spectrum methods cannot guarantee that the solution is always positive, and there will be negative cases at the end of the PDF. Furthermore, the results of the 
comparison of the maximum absolute error when selecting 200 and 1000 calculation points are shown in Fig. 5. It can be found that at 200 points, the super-DL-FP algorithm has an error of a power of $10^{-2}$, while the other three schemes have an error of a power of $10^{-1}$. When the number of calculation points is 1000, the super-DL-FP approach and Chebyshev spectrum method can reach the power order of $10^{-3}$, which is far ahead of the central difference scheme and flow approach.

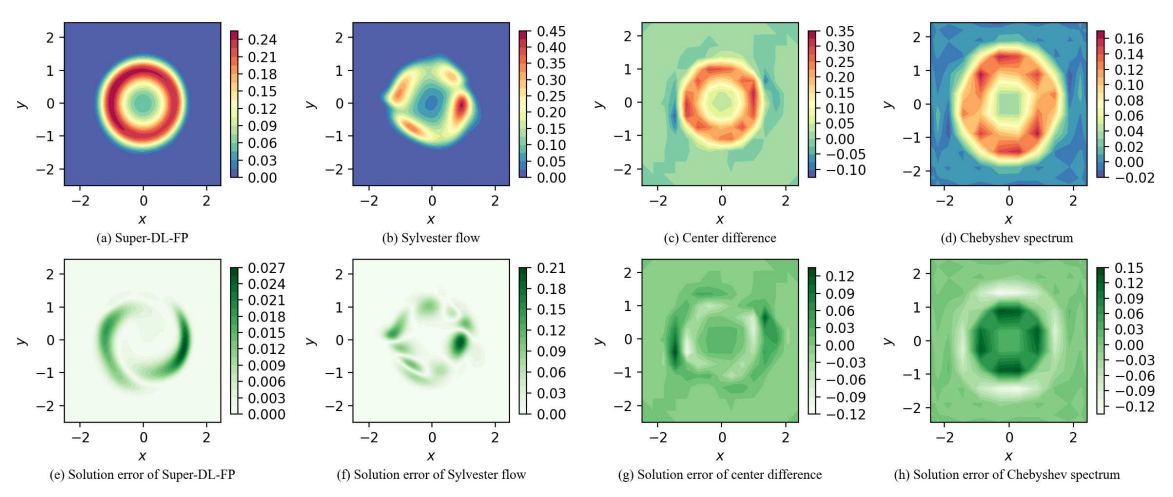

Fig. 4 Calculation results and errors of different algorithms under 200 calculation points with: $\eta=1.0, \sigma^{2}=0.3$.

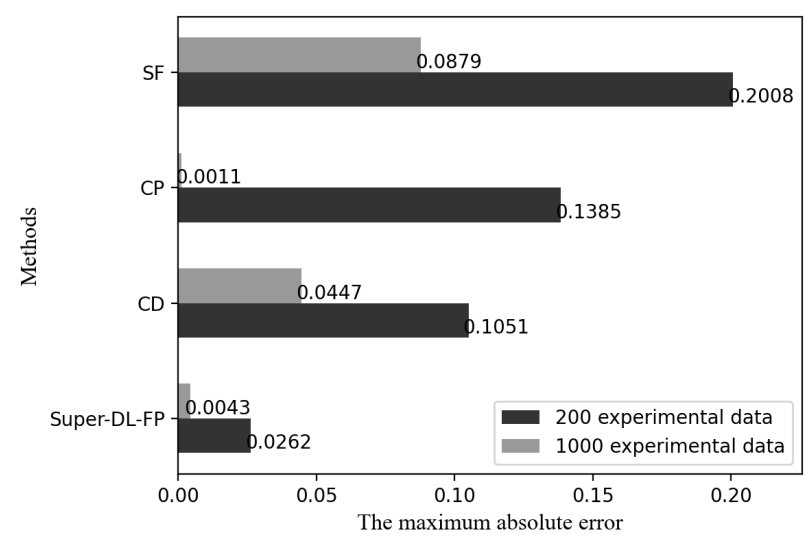

(b) Maximum absolute error comparison between different methods

Fig. 5 Comparison of maximum absolute errors between different algorithms at different number of points. 


\subsection{Example 2}

Consider a 4D system

$$
\left\{\begin{array}{l}
\dot{x}_{1}=x_{3}, \\
\dot{x}_{2}=x_{4}, \\
\dot{x}_{3}=-a x_{3}-\frac{1}{M} \frac{\partial V}{\partial x_{1}}+\sigma_{1} \Gamma, \\
\dot{x}_{4}=-b x_{4}-\frac{1}{I} \frac{\partial V}{\partial x_{2}}+\sigma_{2} \Gamma,
\end{array}\right.
$$

where $V=V\left(x_{1}, x_{2}\right)=k_{1} x_{1}^{2}+k_{2} x_{2}^{2}+\epsilon\left(\lambda_{1} x_{1}^{4}+\lambda_{2} x_{2}^{4}+\mu x_{1}^{2} x_{2}^{2}\right)$ and the corresponding $\mathrm{FP}$ equation is

$-\frac{\partial\left(x_{3} p\right)}{\partial x_{1}}-\frac{\partial\left(x_{4} p\right)}{\partial x_{2}}-\frac{\partial\left[\left(-a x_{3}-\frac{1}{M} \frac{\partial V}{\partial x_{1}} p\right)\right]}{\partial x_{3}}-\frac{\partial\left[\left(-b x_{4}-\frac{1}{I} \frac{\partial V}{\partial x_{2}} p\right)\right]}{\partial x_{4}}+\frac{\sigma_{1}^{2}}{2} \frac{\partial^{2} p}{\partial x_{3}^{2}}+\frac{\sigma_{2}^{2}}{2} \frac{\partial^{2} p}{\partial x_{4}^{2}}=0$.

The exact steady-state solution exists when $\frac{\sigma_{1}^{2} M}{a}=\frac{\sigma_{2}^{2} I}{b}=2 T$ and can be expressed as:

$$
p_{s}=C \cdot \exp \left[-\frac{1}{2 T} V\left(x_{1}, x_{2}\right)-\frac{a}{\sigma_{1}^{2}} x_{3}^{2}-\frac{b}{\sigma_{2}^{2}} x_{4}^{2}\right]
$$

where $C$ is the normalization constant. For the system 14, the calculation interval is selected as $x_{i} \in[-5,5], i=1,2,3,4$. The SRKII is still used to generate random points for Eq. 13 to simulate experimental data, and deep KD-tree algorithm is used to generate different training sets for these random points. In order to study the deep KD-tree, we force the iteration to loop 30 times instead of setting the data ratio coefficient. The results of the utilization rate which is the number of integral data divided by the number of original data, and integral contribution rate of integral points were expected to be analyzed and calculated as shown in Fig. 6. It can be found that the deep KDtree algorithm will reach the point where the utilization rate of integral points is 1 and converge after a finite number of iterations. Besides, there is still a positive correlation between the utilization rate of points and the contribution rate of points, which also confirms the conclusion of the first example. As can be seen from the results in the Fig. 6(a), when the number of experimental points is 100,000 , the integral contribution rate is basically close to 1 , and the convergence is very stable. 

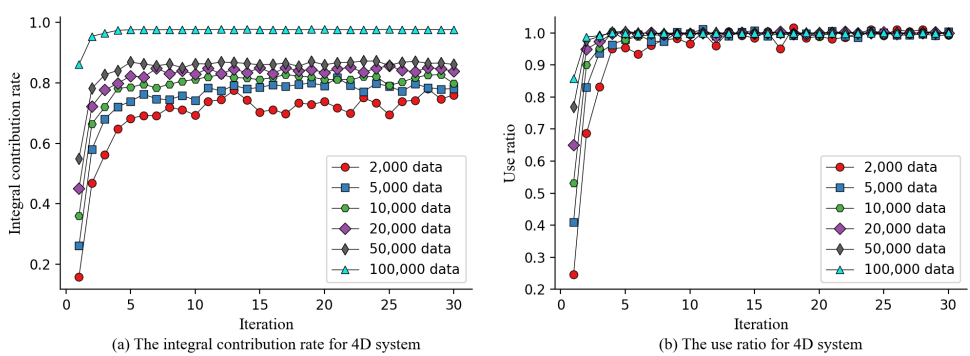

Fig. 6 (a) The utilization rate of different data sets increases with the depth of deep KDtree for system 13. (b) The integral contribution rate of different data sets increases with the depth of deep KD-tree for system 13. The parameters are $a=0.5, b=1, k_{1}=-0.5, \epsilon=$ $0.5, \lambda_{1}=0.25, \lambda_{2}=0.125, \mu=0.375, \sigma_{1}^{2}=2, \sigma_{2}^{2}=4, M=I=1$.

However, we still hope to select a smaller number to complete the test. Here, 20,000 experimental points are selected, and the coefficient of data ratio coefficient is set as 1 . At this time, 19,818 integral points and 8,559 boundary points are obtained, and the integral value of these points is 0.8 . The training points are input into the super DL-FP algorithm to obtain the analytical result with $a_{2}=0.01$. Similarly, the normalized value is calculated by using the Vegas integral. The normalized PDF is obtained and the results are shown in Fig. 7. Fig. 7 shows the two-dimensional PDF, and it can be found that the error of the results is in the power of $10^{-4}$. In this example, we mainly want to emphasize the advantages of our method for the use of small amount of data. The data usage and error of the calculation results in this example are difficult to achieve by using the difference schemes.
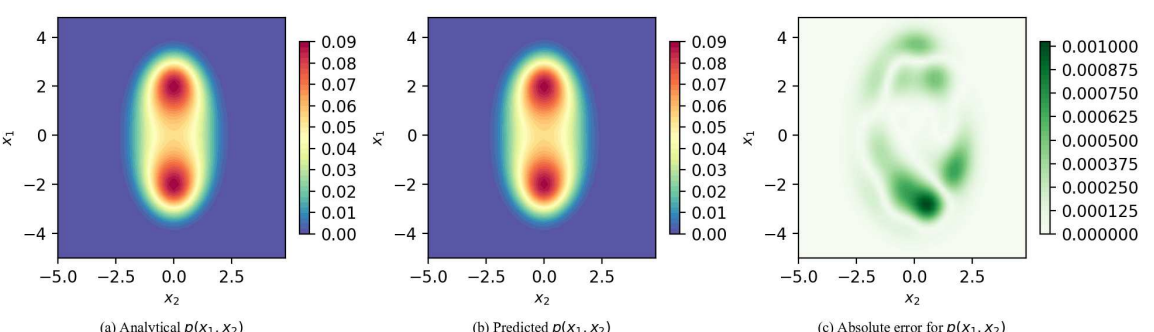

Fig. 7 (a) Analytical $p\left(x_{1}, x_{2}\right)$ for system 13. (b) the predicted $p\left(x_{1}, x_{2}\right)$ with super-DLFP algorithm. (c) The absolute error between the analytical solution and the super-DL-FP solution. The parameters are $a=0.5, b=1, k_{1}=-0.5, \epsilon=0.5, \lambda_{1}=0.25, \lambda_{2}=0.125, \mu=$ $0.375, \sigma_{1}^{2}=2, \sigma_{2}^{2}=4, M=I=1$. 
3.3 Example 3

In this example, a $6 \mathrm{D}$ systems is considered which is

$$
\left\{\begin{array}{l}
\dot{x}_{1}=y_{1} \\
\dot{y}_{1}=-y_{1}-\frac{\partial V}{\partial x_{1}}+\sigma_{1} \Gamma \\
\dot{x}_{2}=y_{2} \\
\dot{y}_{2}=-y_{2}-\frac{\partial V}{\partial x_{2}}+\sigma_{2} \Gamma \\
\dot{x}_{3}=y_{3} \\
\dot{y}_{3}=-y_{3}-\frac{\partial V}{\partial x_{3}}+\sigma_{3} \Gamma
\end{array}\right.
$$

where $V=V\left(x_{1}, x_{2}, x_{3}\right)=\frac{1}{2}\left(x_{1}^{2}+x_{2}^{2}+x_{3}^{2}\right)+\frac{1}{4}\left(x_{1} x_{2}+x_{1} x_{3}\right)$, and the corresponding stationary $\mathrm{FP}$ equation is

$$
\begin{array}{r}
-\frac{\partial\left(y_{1} p\right)}{\partial x_{1}}-\frac{\partial\left(y_{2} p\right)}{\partial x_{2}}-\frac{\partial\left(y_{3} p\right)}{\partial x_{3}}-\frac{\partial\left[\left(-y_{1}-\frac{\partial V}{\partial x_{1}} p\right)\right]}{\partial y_{1}}-\frac{\partial\left[\left(-y_{2}-\frac{\partial V}{\partial x_{2}} p\right)\right]}{\partial y_{2}}- \\
\frac{\partial\left[\left(-y_{3}-\frac{\partial V}{\partial x_{3}} p\right)\right]}{\partial y_{3}}+\frac{\sigma_{1}^{2}}{2} \frac{\partial^{2} p}{\partial y_{1}^{2}}+\frac{\sigma_{2}^{2}}{2} \frac{\partial^{2} p}{\partial y_{2}^{2}}+\frac{\sigma_{3}^{2}}{2} \frac{\partial^{2} p}{\partial y_{3}^{2}}=0 .
\end{array}
$$

Similarly, when $\sigma_{1}^{2}=\sigma_{2}^{2}=\sigma_{3}^{2}=4 T$, the Eq.17 owns the exact stationary solution

$$
p_{s}=C \cdot \exp \left[-\frac{1}{2 T} V\left(x_{1}, x_{2}, x_{3}\right)-\frac{1}{\sigma_{1}^{2}} y_{1}^{2}-\frac{1}{\sigma_{2}^{2}} y_{2}^{2}-\frac{1}{\sigma_{3}^{2}} y_{3}^{2}\right]
$$

where $C$ is the normalization constant. When solving the Eq. 17, the first step is still to obtain the training points which can be done by applying the SRKII method for Eq. 16. In order to further analyze and verify the performance of deep KD-tree, the data ratio coefficient is still not set. Instead, the iteration cycle is forced for 30 times. The results are shown in Fig. 8, which proves once again that the utilization rate of points is close to $100 \%$ and converges after reaching a certain number of cycles. Therefore, it can be found from the analysis of this example and the previous one that in the actual use of deep $\mathrm{KD}$-tree, it is reasonable to set a data ratio coefficient, but it should not be greater than 1. At the same time, the results in the Fig. 8 again show that the utilization rate of integral points is positively correlated with the contribution rate of integral points. When the utilization rate of integral points converges, the contribution rate of integral points also converges. The contribution rate of integral points will increase with the increase of the number of experimental points. 

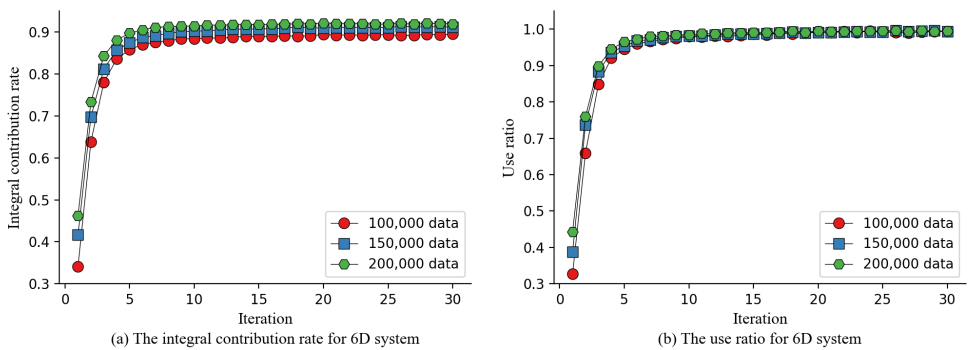

Fig. 8 (a) The utilization rate of different data sets increases with the depth of deep KDtree for system 16. (b) The integral contribution rate of different data sets increases with the depth of deep KD-tree for system 16. The parameters are $\sigma_{1}^{2}=\sigma_{2}^{2}=\sigma_{3}^{2}=4$.

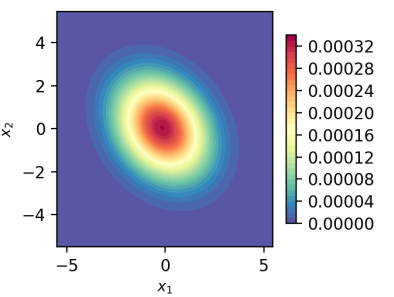

(a) Analytical $p\left(x_{1}, x_{2} \mid y_{1}=x_{3}=y_{2}=y_{3}=0.6\right)$

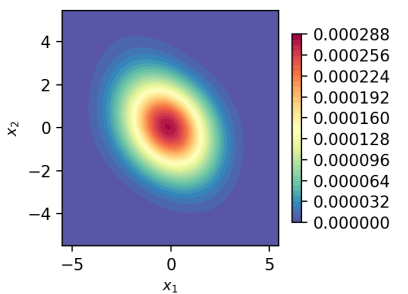

(b) Predicted $p\left(x_{1}, x_{2} \mid y_{1}=x_{3}=y_{2}=y_{3}=0.6\right)$

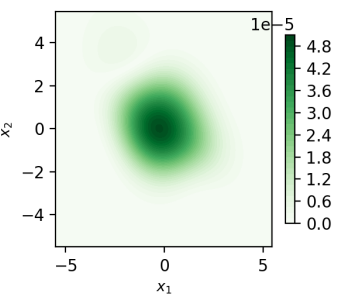

(c) Absolute error between (a) and (b)

Fig. 9 (a) Analytical conditional PDF $p\left(x_{1}, x_{2} \mid y_{1}=x_{3}=y_{2}=y_{3}=0.6\right)$ for system 16. (b) the predicted $p\left(x_{1}, x_{2} \mid y_{1}=x_{3}=y_{2}=y_{3}=0.6\right)$ super-DL-FP solution. (c) The absolute error between the analytical solution and the super-DL-FP solution. The parameters are $\sigma_{1}^{2}=\sigma_{2}^{2}=\sigma_{3}^{2}=4$.

Here we selected 200,000 basic experimental data sets. 175,000 integral data sets and 500,000 boundary sets can be generated after 5 times of deep KD-tree. At this point, it can be found that the number of boundary sets is too large. In this case, a specific gravity can be set and a certain number of boundary sets can be randomly selected according to uniform distribution as the final boundary sets to be put into super-DL-FP. In this example, 20,000 boundary sets are selected. The results in Fig. 9 show the comparison between the conditional PDF calculated using the super-DL-FP and the analytical results. It can be found that the error of the results is relatively small. In particular, the data usage of our approach is very competitive compared with other methods. It still needs to be emphasized that this algorithm can acquire the analytical results, which can be obtained at any point and can also be used for subsequent analytical processing. 


\section{Conclusions}

This paper is mainly concerned with how to solve the FP equation with a small amount of data instead of the whole discrete points. A deep KD-tree method is proposed to estimate the normalization condition in FP equation with random points. The basic idea is to divide the mesh based on random points by an iterative KD-tree algorithm to get the integral volume partition of random points matching. The super-DL-FP algorithm which can solve the FP equations with a small amount of data is proposed through combining the deep KD-tree with the DL-FP framework and its correctness is proved by three numerical examples. We found that: (1) The deep KD-tree method can improve the accuracy of normalized integral estimation, and thus improve the accuracy of training results. (2) The deep KD-tree method can converge after a certain number of iterations. (3) Compared with the traditional difference method and normalized flow approach, the super-DL-FP algorithm has a high precision in solving FP equation with a small amount of data. (4) The superDL-FP framework obtains an analytical solution, which can provide a good foundation for subsequent analytical analysis. Compared with the other NNbased methods for solving differential equation problems, this paper focuses more on how to fully utilize and process data without any increase in any prior condition. We believe that the approach presented in this paper can provide a data processing paradigm for other NN approaches to differential equation problems. The main contribution of this paper is the deep KD-tree algorithm, which can be used to estimate normalized integrals with a small amount of data instead of the total amount. We believe that this method can also provide an idea for other integral-differential equations using the NN method. However, the deep KD-tree approach is only illustrated from the perspective of visualization, without theoretical proof, which is a place that needs to be further improved. At the same time, in terms of the scheme of integral estimation, this paper adopts the more conservative midpoint integral, there may be more appropriate integrals, such as quantile integrals, which also require further study.

\section{Acknowledgements}

This work was partly supported by the NSF of China (No. 12072264), the Key International (Regional) Joint Research Program of the NSF of China (No. 12120101002), the Research Funds for Interdisciplinary Subject of NPU, and the Shaanxi Provincial Key R\&D Program (Grant Nos. 2020KW-013 and 2019TD-010).

\section{Declarations}

Conflict of interest: The authors declare that they have no conflict of interest. 


\section{Data availability}

Data sharing is not applicable to this article as no datasets were generated or analyzed during the current study.

\section{References}

1. Berg, R.v.d., Hasenclever, L., Tomczak, J.M., Welling, M.: Sylvester normalizing flows for variational inference. arXiv preprint arXiv:1803.05649 (2018)

2. Brown, R.A.: Building a balanced $k$-d tree in $O(k n \log n)$ time. Journal of Computer Graphics Techniques Vol 4(1) (2015)

3. Ceccato, A., Frezzato, D.: Remarks on the chemical Fokker-Planck and Langevin equations: Nonphysical currents at equilibrium. Journal of Chemical Physics 148, 064114 (2018)

4. Chen, X., Yang, L., Duan, J., Karniadakis, G.E.: Solving inverse stochastic problems from discrete particle observations using the Fokker-Planck equation and physicsinformed neural networks. SIAM Journal on Scientific Computing 43(3), B811-B830 (2021)

5. Drozdov, A.N.: Accurate path integral representations of the Fokker-Planck equation with a linear reference system: Comparative study of current theories. Physical Review E 57, 146-158 (1998)

6. Escobedo, M., Herrero, M., Velazquez, J.: A nonlinear Fokker-Planck equation modelling the approach to thermal equilibrium in a homogeneous plasma. Transactions of the American Mathematical Society 350(10), 3837-3901 (1998)

7. Foster, T., Lei, C.L., Robinson, M., Gavaghan, D., Lambert, B.: Model evidence with fast tree based quadrature. stat 1050, 22 (2020)

8. Furioli, G., Pulvirenti, A., Terraneo, E., Toscani, G.: Fokker-Planck equations in the modeling of socio-economic phenomena. Mathematical Models \& Methods in Applied Sciences 27, 44 (2016)

9. Galán, R.F., G Bard, E., Urban, N.N.: Stochastic dynamics of uncoupled neural oscillators: Fokker-Planck studies with the finite element method. Physical Review E 76 $056110(2007)$

10. Han, J., Jentzen, A., Weinan, E.: Solving high-dimensional partial differential equations using deep learning. Proceedings of the National Academy of Sciences 115, 8505-8510 (2018)

11. Honeycutt, R.L.: Stochastic Runge-Kutta algorithms. i. white noise. Physical Review A 45, 600 (1992)

12. Jeffrey, N., Wandelt, B.D.: Solving high-dimensional parameter inference: marginal posterior densities \& moment networks. arXiv preprint arXiv:2011.05991 (2020)

13. Jiang, Y.: A new analysis of stability and convergence for finite difference schemes solving the time fractional Fokker-Planck equation. Applied Mathematical Modelling 39, 1163-1171 (2015)

14. Lepage, G.P.: Adaptive multidimensional integration: vegas enhanced. Journal of Computational Physics 439, 110386 (2021)

15. Li, J., Chen, J., Li, B.: Gradient-optimized physics-informed neural networks (GOPINNs): a deep learning method for solving the complex modified KdV equation. Nonlinear Dynamics pp. 1-12 (2021)

16. Ma, J., Xu, Y., Li, Y., Tian, R., Chen, G., Kurths, J.: Precursor criteria for noiseinduced critical transitions in multi-stable systems. Nonlinear Dynamics 101(1), 21-35 (2020)

17. Náprstek, J., Král, R.: Finite element method analysis of Fokker-Planck equation in stationary and evolutionary versions. Advances in Engineering Software 72, 28-38 (2014)

18. Raissi, M., Perdikaris, P., Karniadakis, G.E.: Physics-informed neural networks: A deep learning framework for solving forward and inverse problems involving nonlinear partial differential equations. Journal of Computational Physics 378, 686-707 (2019) 
19. Risken, H.: Fokker-Planck equation. In: The Fokker-Planck Equation, pp. 63-95. Springer (1996)

20. Sepehrian, B., Radpoor, M.K.: Numerical solution of non-linear Fokker-Planck equation using finite differences method and the cubic spline functions. Applied Mathematics \& Computation 262, 187-190 (2015)

21. Sirignano, J., Spiliopoulos, K.: DGM: A deep learning algorithm for solving partial differential equations. Journal of Computational Physics 375, 1339-1364 (2018)

22. Wang, Z., Xu, Y., Li, Y., Kurths, J.: $\alpha$-stable noise-induced coherence on a spatially extended Fitzhugh Nagumo system. Journal of Statistical Mechanics: Theory and Experiment 2019(10), 103501 (2019)

23. Xiao, L.: A finite-time recurrent neural network for solving online time-varying Sylvester matrix equation based on a new evolution formula. Nonlinear Dynamics 90(3), 1581$1591(2017)$

24. Xu, Y., Li, Y., Zhang, H., Li, X., Kurths, J.: The switch in a genetic toggle system with Lévy noise. Scientific Reports 6(1), 31505-31505 (2016)

25. Xu, Y., Liu, Q., Guo, G., Xu, C., Liu, D.: Dynamical responses of airfoil models with harmonic excitation under uncertain disturbance. Nonlinear Dynamics 89(3), 1579$1590(2017)$

26. Xu, Y., Zan, W., Jia, W., Kurths, J.: Path integral solutions of the governing equation of SDEs excited by Lévy white noise. Journal of Computational Physics 394, 41-55 (2019)

27. Xu, Y., Zhang, H., Li, Y., Zhou, K., Liu, Q., Kurths, J.: Solving Fokker-Planck equation using deep learning. Chaos 30(1), 013133 (2020)

28. Zhai, J., Dobson, M., Li, Y.: A deep learning method for solving Fokker-Planck equations. arXiv preprint arXiv:2012.10696 (2020)

29. Zhang, H., Xu, Y., Li, Y., Kurths, J.: Statistical solution to SDEs with $\alpha$-stable Lévy noise via deep neural network. International Journal of Dynamics and Control 8(4), 1129-1140 (2020)

30. Zhang, R.F., Bilige, S.: Bilinear neural network method to obtain the exact analytical solutions of nonlinear partial differential equations and its application to p-gBKP equation. Nonlinear Dynamics 95(4), 3041-3048 (2019)

31. Zhang, X., Xu, Y., Liu, Q., Kurths, J., Grebogi, C.: Rate-dependent bifurcation dodging in a thermoacoustic system driven by colored noise. Nonlinear Dynamics 104(3), 27332743 (2021) 